\title{
The Predictors of Target Lesion Revascularization and Rate of In-Stent Restenosis in the Second-Generation Drug-Eluting Stent Era
}

\author{
Chengbin Zheng, Jeehoon Kang, Kyung Woo Park, Jung-Kyu Han, Han-Mo Yang, \\ Hyun-Jae Kang, Bon-Kwon Koo, and Hyo-Soo Kim \\ Department of Internal Medicine and Cardiovascular Center, Seoul National University Hospital, Seoul, Republic of Korea \\ Correspondence should be addressed to Hyo-Soo Kim; hyosoo@snu.ac.kr
}

Received 31 December 2018; Revised 27 April 2019; Accepted 12 June 2019; Published 1 July 2019

Academic Editor: Andrea Rubboli

Copyright (c) 2019 Chengbin Zheng et al. This is an open access article distributed under the Creative Commons Attribution License, which permits unrestricted use, distribution, and reproduction in any medium, provided the original work is properly cited.

\begin{abstract}
Objectives. The aim of our study was to investigate the predictors of target lesion revascularization (TLR) and to compare the instent restenosis (ISR) progression rates of different 2nd-generation drug-eluting stents (DES). Background. The predictors of early and late TLR after 2nd-generation DES implantation have not been fully evaluated. Methods. We analyzed 944 stented lesions from 394 patients who had at least two serial follow-up angiograms, using quantitative coronary angiography (QCA) analysis. The study endpoints were TLR and the velocity of diameter stenosis (DS) progression. Results. TLR occurred in 58 lesions (6.1\%) during the first angiographic follow-up period and 23 de novo lesions (2.4\%) during the following second interval. Independent predictors for early TLR were diabetes mellitus (DM) (HR 2.58, 95\% CI 1.29-5.15, $\mathrm{p}=0.007)$, previous percutaneous coronary intervention (PCI) (HR 2.41, 95\% CI 1.03-5.65, p=0.043), and postprocedure DS\% (HR 1.08, 95\% CI 1.05-1.11, p <0.001, per 1\%), while predictors of late TLR were previous PCI (HR 9.43, 95\% CI 2.58-34.52, $\mathrm{p}=0.001$ ) and serum C-reactive protein (CRP) (HR 1.60, 95\% CI 1.28-2.00, $\mathrm{p}<0.001$ ). The ISR progression velocity (by DS\%) was $12.1 \pm 21.0 \% /$ year and $3.7 \pm 10.1 \%$ /year during the first and second follow-up periods, respectively, which had no significant difference $(\mathrm{p}>0.05)$ between the four types of DESs. Conclusions. Our data showed that predictors for TLR may be different at different time intervals. DM, pervious PCI, and postprocedure DS could predict early TLR, while previous PCI and CRP level could predict late TLR. Contemporary DESs had similar rates of ISR progression rates. Trial Registration. This study was retrospectively registered and approved by the institutional review board of Seoul National University Hospital (no. 1801-138-918).
\end{abstract}

\section{Introduction}

In-stent restenosis (ISR), which is thought to be mostly caused by neointimal hyperplasia (NIH), was an important medical problem in the era of bare-metal stents (BMS) [1]. Subsequent intravascular ultrasound (IVUS) and quantitative coronary angiography (QCA) studies further strengthened the view that the main mechanism of ISR after BMS implantation was intrastent $\mathrm{NIH}[2,3]$ with a biphasic change of lumen loss in the first 6 months and NIH regression between 6 months and 1-3 years after BMS implantation $[4,5]$. Therefore, late TLR after BMS implantation was not a common phenomenon.
Compared to BMS, DES significantly reduced the rates of ISR and TLR [6, 7]. However, some studies mentioned a "late catch-up" phenomenon after the 1st generation DES implantation [8-14]. The "late catch-up" phenomenon suggests that the mechanism and rate of neointimal formation may be different at different time intervals. Thus, we can assume that the predictors of TLR may also vary from time to time. One clinical study suggested that the risk factors for late TLR were similar to those of early TLR [15]. However, this study only included patients who received sirolimus eluting stent (SES) implantation and was limited by the profound selection bias that favored the use of BMS [16]. At present, there are few studies on predictors of both early and late TLR based on 
contemporary 2nd generation DESs; moreover, the predictors are not consistent.

In this study, we analyzed stented lesions treated with four types of contemporary 2nd generation DESs, using longitudinal QCA analysis. We observed the progression of stented lesions and identified the independent predictors of early and late TLR. Additionally, we compared the ISR progression rates of the four types of 2 nd generation DESs.

\section{Methods}

2.1. Study Design and Population. We enrolled patients who underwent previous percutaneous coronary intervention (PCI) in our institute from serial stent registries. These registries included four types of 2 nd generation DESs: cobalt chromium everolimus-eluting stent (CoCrEES), platinum chromium everolimus-eluting stent ( $\mathrm{PtCr}$ EES), zotarolimus-eluting stent (ZES), and Biolimus-eluting stent (BES). Follow-up coronary angiography (CAG) was recommended at 9 to 12 months after PCI according to the protocol of the individual registries (this is not mandatory). In the patients who consented to and received the first follow-up angiogram, second follow-up angiogram was recommended at 24 months. During the study period (July 2008 to March 2013), 3,365 patients were enrolled in various stent registries in Seoul National University Hospital. Among these population, 3170 patients $(94.2 \%)$ were treated with $2^{\text {nd }}$ generation DESs and 1545 patients (45.9\%) received at least two serial angiographic follow-ups. 944 lesions from 394 patients $(11.7 \%)$ were performed in longitudinal QCA analysis. We performed a sensitivity analysis to check the possibility of selection bias, as in the following table, showing that the baseline demographics were similar between the entire parent population and our study population (Supplementary Table 1). Finally, 394 patients and 944 lesions were analyzed. The study flowchart is shown in Figure 1. 58 lesions in 40 patients were needed for TLR at the first angiographic follow-up (early TLR: $6.1 \%, 10.2 \%)$. And 23 lesions in 19 patients which are non-TLR at the first follow-up are needed for late TLR at the second angiographic follow-up (late TLR: $2.4 \%, 4.8 \%$ ) (Figure 2). The study was approved by the ethics committee and institutional review board and was conducted according to the principles of the Declaration of Helsinki. All patients provided written, informed consent for participation in the registry.

2.2. Procedure and Data Collection. PCI procedure was performed with standard methods. Aspirin 300mg and clopidogrel $300-600 \mathrm{mg}$ were administered to all patients before intervention and all patients were given unfractionated heparin to achieve an activated clotting time of 250 seconds or more. The drugs, devices, and intervention techniques (i.e., usage of glycoprotein IIb/IIIa inhibitors, predilatation devices, type of DES, stenting techniques, and imaging guidance) used during the procedure were all determined by experienced operators. Dual antiplatelet therapy was recommended for all patients after DES implantation.
Clinical and procedural data were collected by clinical research coordinators who were unaware of the purpose of the study. Angiographic images at baseline and two serial follow-up periods saved in DICOM format for review and further analyses.

2.3. Quantitative Coronary Angiography. Quantitative analysis was performed by specialized QCA technicians who were unaware of the purpose of this study and the type of stent used for treatment at the Seoul National University Hospital Cardiovascular Clinical Research Center Angiographic Core Lab. The parameters of angiography were measured using the CAAS II QCA system (Pie Medical, Maastricht, the Netherlands). After calibration with the guiding catheter, we measured the reference vessel diameter and the minimal luminal diameter (MLD) both in-stent and in-segment at baseline and two serial angiographic follow-ups. All measurements were performed on angiograms recorded after intracoronary administration of nitroglycerine.

2.4. Study Endpoints and Definitions. In our study, major adverse cardiac events (MACE) were defined as a composite of all cause death, any myocardial infarction, TLR, and stent thrombosis. TLR was defined as any repeat percutaneous intervention of the target lesion or bypass surgery of the target vessel performed for restenosis or other complication of the target lesion. In this cohort, TLR was performed if angiography during follow-up showed a diameter stenosis $\geq 50 \%$ with at least one of the following: (1) history of recurrent angina pectoris, presumably related to the target vessel; (2) objective signs of ischemia at rest or during exercise test by electrocardiogram, presumably related to target vessel; (3) abnormal test results of invasive functional diagnostic test (fractional flow reserve); or (4) a TLR with a diameter stenosis $\geq 70 \%$, even in the absence of the aforementioned ischemic signs or symptoms. The study endpoints were independent predictors of TLR (early TLR, defined as TLR during the first follow-up period, and late TLR defined as TLR during the period from the first follow-up to the second followup) and the velocity of DS\% progression during the followup periods. TLR was defined as repeat PCI within the stent or the $5 \mathrm{~mm}$ borders proximal or distal to the stent. DS\% was defined as $100 \%$ minus the ratio of MLD and diameter of the reference segment. Early delta DS\% indicated the difference in DS\% between angiogram immediately after index procedure and the first follow-up angiogram, and delayed delta DS\% denoted the changes of DS\% between the first and second follow-up. Depending on the time interval, we converted early and delayed delta DS\% into rates (early DS\%/year \& delayed DS\%/year). All measurements were performed for both in-stent and in-segment.

2.5. Statistical Analysis. Continuous variables are expressed as mean \pm standard deviation. Student's t-test or one-way analysis of variance was used for comparison of continuous variables, and we analyzed categorical variables using the chisquare test (or the Fisher exact test when any expected count was $<5$ for a $2 \times 2$ table) test. To determine the independent 


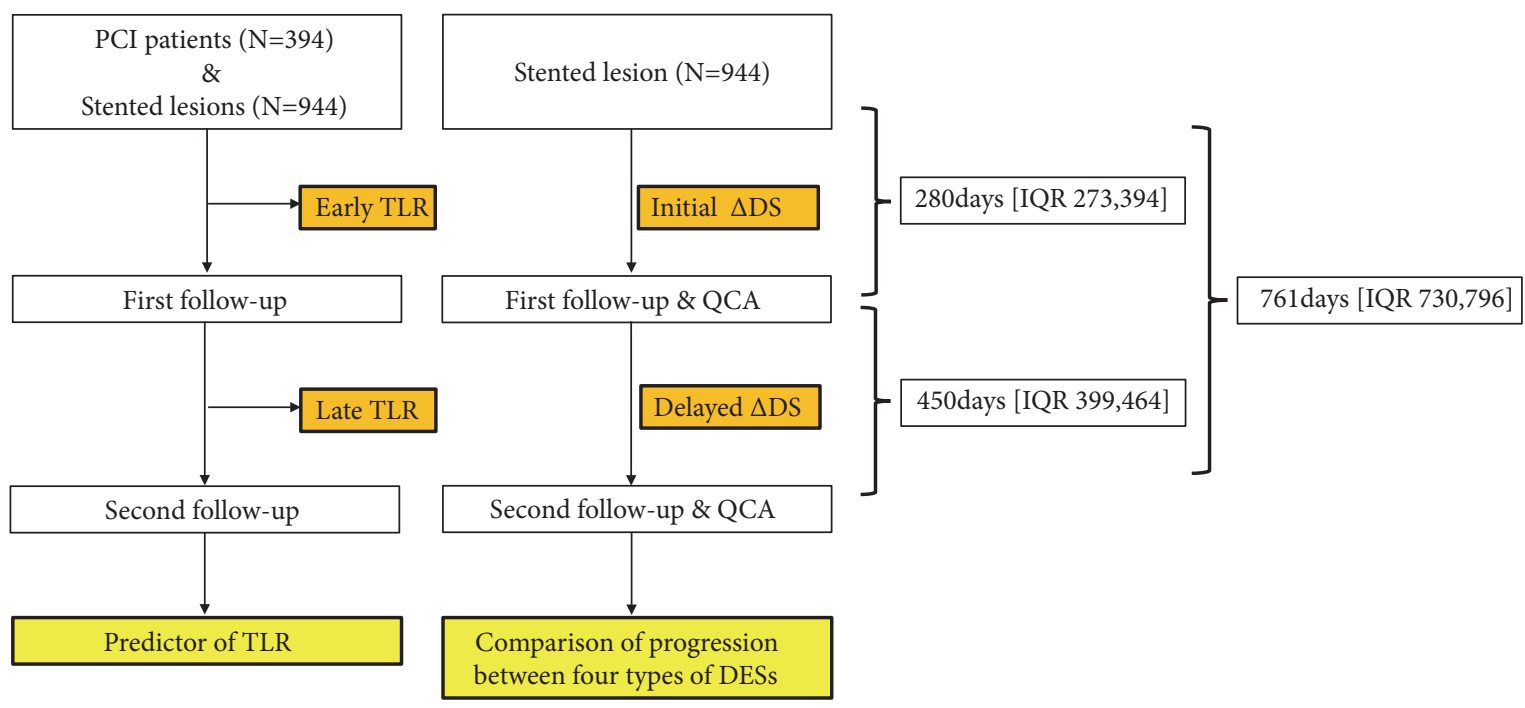

FIgURE 1: Flowchart of the study.

$<$ TLR according to patient $>$

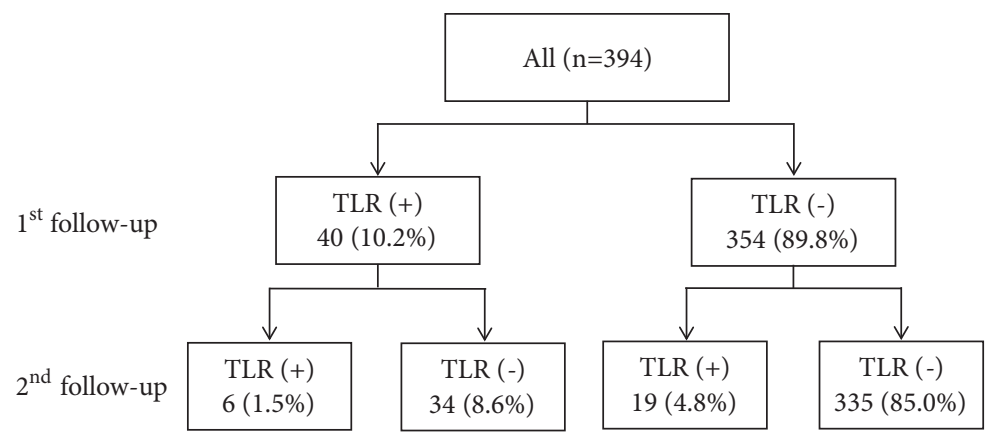

$<$ TLR according to lesion $>$

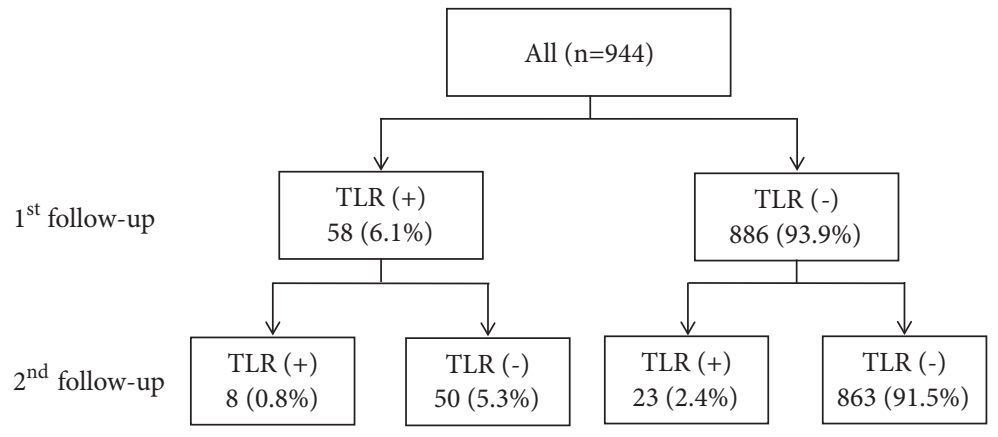

FIGURE 2: Incidence of target lesion revascularization.

predictors of early and late TLR, a Cox proportional hazard model was used. Variables included in a Cox multivariate regression analysis were age, sex, body mass index, HTN, DM, dyslipidemia, current smoking, family history of coronary artery disease, previous PCI, chronic renal failure (creatinine clearance level $<60 \mathrm{ml} / \mathrm{min}$, stage 3 or higher), multivessel disease, left ventricular dysfunction, hemoglobin, CRP as clinical factors and lesion type B2/C, reference vessel diameter, lesion length, preprocedure DS\%, and postprocedure DS\% in-stent as lesion-related factors. Statistical analysis was performed with SPSS (version 23.0), and a value of $P$ values less than 0.05 was considered statistically significant. 


\section{Results}

From July 2008 to March 2013, a total of 394 patients with 944 lesions were enrolled in the study. The mean follow-up duration from baseline to the first and second angiography was $325 \pm 90$ days and $772 \pm 133$ days, respectively. The study flowchart is shown in Figure 1.

3.1. Baseline Characteristics. The mean age of our study population was $65.5 \pm 10.4$ years, $73.4 \%$ were male patients, $69.8 \%$ had hypertension (HTN), and $33.8 \%$ had DM. The mean reference vessel diameter of the lesions was $2.92 \pm 0.52$ $\mathrm{mm}$ and mean length of lesions was $27.7 \pm 17.3 \mathrm{~mm}$. Baseline patient and lesion characteristics of the study population are summarized in Supplementary Table 2. Laboratory findings at the follow-up periods are shown in Supplementary Table 3.

3.2. Incidence and Predictors of Target Lesion Revascularization. The incidence of all events is shown in Supplementary Table 4. The cumulative incidence of TLR is shown in Figure 2. TLR was performed for 58 lesions (6.1\%) in 40 patients $(10.2 \%)$ at the first angiographic follow-up period and for 23 lesions $(2.4 \%)$ in 19 patients $(4.8 \%)$ at the second follow-up period. TLR in this cohort included ischemic driven TLR and TLR during routine angiographic followup. Ischemia driven TLR accounted for $15.5 \%$ and $30.4 \%$ at 1st follow-up and 2nd follow-up, respectively (Supplementary Table 5), and information on TLR methods was described in Supplementary Table 6. In the baseline characteristics, previous PCI and chronic renal failure were more common in patients who experienced early TLR; the hemoglobin level was lower in these patients. Lesion characteristics showed that postprocedural MLD was smaller and postprocedural DS\% was larger in the early TLR group (Table 1). However, no discrepant factors were found between the late TLR and nonlate TLR group (Table 2). Regarding independent predictors of TLR, multivariate analysis results showed chronic renal failure (HR 2.37, 95\% CI 0.94-5.98, $\mathrm{p}=0.068$ ) and hemoglobin (HR 0.94, 95\% CI 0.77-1.16, $\mathrm{p}=0.586$ ) could not predict early TLR, although significant differences were shown in the baseline analysis. However, DM (HR 2.58, 95\% CI 1.29-5.15, $\mathrm{p}=0.007$ ), previous PCI (HR: 2.41, 95\% CI 1.03-5.65, $\mathrm{p}=0.043$ ), and postprocedure DS\% (HR 1.08, 95\% CI 1.05-1.11, p<0.001, per DS of $1 \%$ ) were independent predictors of early TLR (Table 3 ). Conversely, independent predictors of late TLR included previous PCI (HR 9.43, 95\% CI 2.58-34.52, $\mathrm{p}=0.001)$ and CRP (HR 1.60, 95\% CI 1.28-2.00, $\mathrm{p}<0.001$ ) (Table 4).

3.3. Angiographic Outcome. In our study, 944 lesions were treated using four types of 2nd generation DES: CoCr-EES, ZES, BES, and PtCr-EES; each accounted for $25.8 \%, 32.8 \%$, $17.6 \%$, and $23.7 \%$, respectively. There were no significant differences in both reference vessel diameter $(\mathrm{p}=0.054$ using ANOVA) and lesion length ( $\mathrm{p}=0.247$ using ANOVA) between the four types of DES. QCA analysis of 944 lesions showed that the mean angiographic DS\% before the procedure was
$74.8 \% \pm 15.7 \%$; it decreased to $11.8 \% \pm 8.5 \%$ (in-stent DS\%) and $21.5 \% \pm 11.6 \%$ (in-segment DS\%) after the procedure (Supplementary Table 2). The mean MLD also showed no significant difference between the four DES groups at all the different points in time (initial state, postprocedure, at the first angiographic follow-up, and at the second angiographic follow-up) (Table 5). The MLD cumulative probability curve of the four groups is shown in Supplementary Figure 1. Both early delta DS\% and delayed delta DS\% were also similar between the four DESs (Early delta DS\%: $9.5 \pm 16.0 \%, 10.3$ $\pm 15.2 \%, 9.5 \pm 14.4 \%, 11.7 \pm 17.6 \%, \mathrm{p}=0.204$ using ANOVA, delayed delta DS\%: $4.8 \pm 14.1 \%, 3.7 \pm 9.9 \%, 5.3 \pm 11.5 \%, 5.1$ $\pm 11.1 \%$, $\mathrm{p}=0.486$ using ANOVA, for CoCr-EES, ZES, BES, and PtCr-EES, respectively.)

Regarding ISR progression, the early delta DS\%/year was $12.12 \% \pm 20.97 \% / y e a r$, and delayed delta DS\%/year was $3.68 \% \pm 10.10 \% /$ year, showing that the delayed ISR progression rate was about $30 \%$ of the early progression rate. Between the four types of DES, both the early ISR progression rate and delayed ISR progression rate showed no significant difference (early ISR progression rate: $12.2 \% \pm 20.9 \% /$ year, $12.2 \% \pm 18.6 \% /$ year, $14.3 \% \pm 29.8 \% /$ year, $10.4 \% \pm 15.7 \% /$ year, $\mathrm{p}=0.525$; delayed ISR progression rate: $4.1 \% \pm 12.8 \% /$ year, $2.4 \% \pm 8.1 \%$ /year, $4.0 \% \pm 8.3 \% /$ year, $\quad 4.8 \% \pm 10.5 \% /$ year, $\mathrm{p}=0.205)$ (Table 5).

\section{Discussion}

In this study, the cumulative incidence rate for early TLR was $6.1 \%$, and $2.4 \%$ for late TLR in stented lesions, during the overall median follow-up period of 772 days. The rate of ISR progression of early and late was $12.1 \pm 21.0 \% /$ year and $3.7 \pm 10.1 \%$ /year, respectively. Additionally, independent predictors of early TLR were DM, previous PCI, and postprocedure DS\%, while those for late TLR were previous PCI and high serum CRP level, differing from each other. Additional analysis showed that the rate of early DS\%/year and delayed DS\%/year were all similar ( $p>0.05)$ among the four types of DES.

4.1. Mechanism of Restenosis after DES Implantation. Restenosis is a progressive phenomenon and the specific mechanism at different time intervals of ISR is difficult to explain exactly. Since entering the era of support, the two mechanisms recoil and vascular remodeling had been almost cancelled with the advent of stents compared to simple balloon angioplasty and NIH became the primary mechanism for restenosis [17]. ISR is primarily a nonspecific inflammatory response to vessel wall injury and the injured tissue reacts via an inflammatory process that leads to $\mathrm{NIH}$, eventually leading to lumen narrowing. Regardless of the exact pathophysiology, ISR is the end result of endothelial injury caused by stent deployment and foreign materials left at the deployment site [16,18-20]. Goto K et al. retrospectively analyzed 298 ISR lesions using IVUS data. The main findings of this study were that NIH was an important mechanism of ISR even in the 2 nd generation DES era [21]. Compared with BMS, although the drug and polymer of DES counteract the 
TABLE 1: Baseline patients and lesion characteristics according to early TLR.

\begin{tabular}{|c|c|c|c|}
\hline Clinical Factors & $\begin{array}{l}\text { Early TLR(-) } \\
\text { (354 patients) }\end{array}$ & $\begin{array}{c}\text { Early TLR(+) } \\
\text { (40 patients) }\end{array}$ & $\mathrm{P}$ \\
\hline Age (years old) & $65.3 \pm 10.4$ & $66.9 \pm 11.0$ & 0.369 \\
\hline BMI $\left(\mathrm{kg} / \mathrm{m}^{2}\right)$ & $24.8 \pm 2.9$ & $23.9 \pm 3.3$ & 0.073 \\
\hline Male sex, n (\%) & $265(74.9)$ & $24(60.0)$ & 0.068 \\
\hline Previous PCI, n (\%) & $38(10.7)$ & $10(25.0)$ & 0.018 \\
\hline Diabetes mellitus, n (\%) & $114(32.2)$ & $19(47.5)$ & 0.078 \\
\hline Hypertension, n (\%) & $246(69.5)$ & $29(72.5)$ & 0.833 \\
\hline CRF, n (\%) & $137(38.7)$ & $25(62.5)$ & 0.006 \\
\hline Dyslipidemia, n (\%) & $225(63.6)$ & $23(57.5)$ & 0.562 \\
\hline Current smoking, n (\%) & $95(26.8)$ & $9(22.5)$ & 0.689 \\
\hline FHx of CAD, n (\%) & $44(12.4)$ & $6(15.0)$ & 0.807 \\
\hline LV ejection fraction (\%) & $60.3 \pm 8.3$ & $57.3 \pm 10.6$ & 0.119 \\
\hline Clinical diagnosis & & & 0.127 \\
\hline (i) Silent ischemia, n (\%) & $21(5.9)$ & $0(0)$ & \\
\hline (ii) Stable angina, $\mathrm{n}(\%)$ & $209(59.0)$ & $23(57.5)$ & \\
\hline (iii) Unstable angina, $\mathrm{n}(\%)$ & $71(20.1)$ & $7(17.5)$ & \\
\hline (iv) STEMI, n (\%) & $22(6.2)$ & $2(5.0)$ & \\
\hline (v) NSTEMI, n (\%) & $31(8.8)$ & $8(20.0)$ & \\
\hline Multi Vessel disease, n (\%) & $255(72.0)$ & $31(77.5)$ & 0.576 \\
\hline \multicolumn{4}{|l|}{ Laboratory tests } \\
\hline $\mathrm{WBC}\left(10^{9} / \mathrm{L}\right)$ & $6.9 \pm 2.4$ & $6.5 \pm 1.7$ & 0.332 \\
\hline Hemoglobin (g/dl) & $13.6 \pm 1.8$ & $12.9 \pm 2.0$ & 0.018 \\
\hline Creatinine $(\mathrm{mg} / \mathrm{dl})$ & $1.05 \pm 0.44$ & $1.39 \pm 1.60$ & 0.183 \\
\hline $\operatorname{GFR}\left(\mathrm{ml} / \mathrm{min} / 1.73 \mathrm{~m}^{2}\right)$ & $70.8 \pm 24.0$ & $57.5 \pm 28.2$ & 0.015 \\
\hline Total cholesterol (mg/dl) & $157 \pm 38$ & $159 \pm 45$ & 0.845 \\
\hline Triglyceride (mg/dl) & $140 \pm 86$ & $120 \pm 66$ & 0.193 \\
\hline $\mathrm{LDL}(\mathrm{mg} / \mathrm{dl})$ & $97 \pm 35$ & $94 \pm 44$ & 0.666 \\
\hline HDL (mg/dl) & $42 \pm 11$ & $44 \pm 12$ & 0.302 \\
\hline CRP (mg/dl) & $0.40 \pm 1.17$ & $0.37 \pm 0.84$ & 0.870 \\
\hline \multicolumn{4}{|l|}{ Medication at discharge } \\
\hline (i) Aspirin, n (\%) & $353(99.7)$ & $40(100.0)$ & 1.000 \\
\hline (ii) Clopidogrel, n (\%) & $353(99.7)$ & $40(100.0)$ & 1.000 \\
\hline (iii) DAPT, $\mathrm{n}(\%)^{\#}$ & $352(99.4)$ & $40(100.0)$ & 1.000 \\
\hline (iv) Beta blocker, n (\%) & $251(70.9)$ & $28(70.0)$ & 1.000 \\
\hline (v) Statin, n (\%) & $337(95.2)$ & $39(97.5)$ & 1.000 \\
\hline (vi) CCB, n (\%) & $75(21.2)$ & $11(27.5)$ & 0.418 \\
\hline (vii) ACEI, n (\%) & $85(24.0)$ & $16(40.0)$ & 0.035 \\
\hline (viii) ARB, n (\%) & $134(37.9)$ & $19(47.5)$ & 0.237 \\
\hline \multicolumn{4}{|l|}{ Medication at first follow-up } \\
\hline (i) Aspirin, n (\%) & $351(99.2)$ & $40(100.0)$ & 1.000 \\
\hline (ii) Clopidogrel, n (\%) & $346(97.7)$ & $39(97.5)$ & 1.000 \\
\hline (iii) DAPT, n $(\%)^{\#}$ & $343(96.9)$ & $39(97.5)$ & 1.000 \\
\hline (iv) Beta blocker, n (\%) & $266(75.1)$ & $28(70.0)$ & 0.451 \\
\hline (v) Statin, n (\%) & $352(99.4)$ & $40(100.0)$ & 1.000 \\
\hline (vi) CCB, n (\%) & $79(22.3)$ & $8(20.0)$ & 0.842 \\
\hline (vii) ACEI, n (\%) & $36(10.2)$ & $8(20.0)$ & 0.106 \\
\hline (viii) ARB, n (\%) & $145(41.0)$ & $13(32.5)$ & 0.395 \\
\hline \multicolumn{4}{|l|}{ Medication at second follow-up } \\
\hline (i) Aspirin, n (\%) & $324(91.5)$ & $38(95.0)$ & 0.758 \\
\hline (ii) Clopidogrel, n (\%) & $300(84.7)$ & $37(92.5)$ & 0.239 \\
\hline (iii) DAPT, $\mathrm{n}(\%)^{\#}$ & $272(76.8)$ & $35(87.5)$ & 0.159 \\
\hline
\end{tabular}


TABle 1: Continued.

\begin{tabular}{|c|c|c|c|}
\hline Clinical Factors & $\begin{array}{l}\text { Early TLR(-) } \\
\text { (354 patients) }\end{array}$ & $\begin{array}{c}\text { Early TLR(+) } \\
\text { (40 patients) }\end{array}$ & $\mathrm{P}$ \\
\hline (iv) Beta blocker, n (\%) & $256(72.3)$ & $25(62.5)$ & 0.200 \\
\hline (v) Statin, n (\%) & $349(98.6)$ & $39(97.5)$ & 0.476 \\
\hline (vi) CCB, n (\%) & $68(19.2)$ & $9(22.5)$ & 0.674 \\
\hline (vii) ACEI, n (\%) & $28(7.9)$ & $7(17.5)$ & 0.070 \\
\hline (viii) ARB, n (\%) & $137(38.7)$ & $13(32.5)$ & 0.495 \\
\hline \multirow[t]{2}{*}{ Lesion factors } & Early TLR(-) & Early TLR(+) & \\
\hline & (886 lesions) & (58 lesions) & \\
\hline Treated coronary location & & & 0.854 \\
\hline (i) LAD, n (\%) & $411(46.4)$ & $28(48.3)$ & \\
\hline (ii) LCX, n (\%) & $218(24.6)$ & $16(27.6)$ & \\
\hline (iii) RCA, n (\%) & $255(28.8)$ & $14(24.1)$ & \\
\hline Bifurcation lesion, n (\%) & $308(34.8)$ & $25(43.1)$ & 0.204 \\
\hline Calcified lesion & & & 0.004 \\
\hline (i) None, n (\%) & $451(51.2)$ & $26(44.8)$ & \\
\hline (ii) Mild, n (\%) & $155(17.6)$ & $4(6.9)$ & \\
\hline (iii) Moderate, n (\%) & $114(12.9)$ & $7(12.1)$ & \\
\hline (iv) Severe, $\mathrm{n}(\%)$ & $161(18.3)$ & $21(36.2)$ & \\
\hline Tortuous lesion & & & 0.465 \\
\hline (i) None, n (\%) & $819(92.9)$ & $56(96.6)$ & \\
\hline (ii) Mild, n (\%) & $24(2.7)$ & $0(0)$ & \\
\hline (iii) Moderate, n (\%) & $26(2.9)$ & $2(3.4)$ & \\
\hline (iv) Severe, n (\%) & $13(1.5)$ & $0(0)$ & \\
\hline Angulation lesion, n (\%) & & & 0.305 \\
\hline (i) None $\left(<45^{\circ}\right), \mathrm{n}(\%)$ & $821(93.3)$ & $56(98.2)$ & \\
\hline (ii) Moderate $\left(45^{\circ}<90^{\circ}\right), \mathrm{n}(\%)$ & $37(4.2)$ & $1(1.8)$ & \\
\hline (iii) Extreme n $\left(>90^{\circ}\right), \mathrm{n}(\%)$ & $22(2.5)$ & $0(0)$ & \\
\hline Thrombus in lesion, n (\%) & $29(3.3)$ & $0(0)$ & 0.291 \\
\hline Ostial lesion, n (\%) & $169(19.1)$ & $14(24.1)$ & 0.390 \\
\hline Ulceration, n (\%) & $2(0.2)$ & $3(5.2)$ & 0.002 \\
\hline Aneurysm, n (\%) & $10(1.1)$ & $0(0)$ & 1.000 \\
\hline Lesion type & & & 0.142 \\
\hline (i) A, n (\%) & $92(10.4)$ & $8(13.8)$ & \\
\hline (ii) B1, n (\%) & $214(24.2)$ & $8(13.8)$ & \\
\hline (iii) $\mathrm{B} 2, \mathrm{n}(\%)$ & $52(5.9)$ & $7(12.1)$ & \\
\hline (iv) C, n (\%) & $499(56.3)$ & $34(58.6)$ & \\
\hline Type B2/C lesion, n (\%) & $551(64.3)$ & $41(71.9)$ & 0.305 \\
\hline Reference vessel diameter (mm) & $2.92 \pm 0.51$ & $2.78 \pm 0.66$ & 0.100 \\
\hline Lesion length (mm) & $27.7 \pm 17.1$ & $28.8 \pm 19.4$ & 0.647 \\
\hline Pre-procedure MLD (mm) & $0.74 \pm 0.50$ & $0.68 \pm 0.49$ & 0.385 \\
\hline Pre-procedure DS (\%) & $74.7 \pm 15.7$ & $75.9 \pm 15.5$ & 0.569 \\
\hline Post-procedure MLD, in- stent (mm) & $2.49 \pm 0.43$ & $2.26 \pm 0.48$ & $<0.001$ \\
\hline Post-procedure MLD, in-segment (mm) & $2.13 \pm 0.51$ & $1.85 \pm 0.57$ & $<0.001$ \\
\hline Post-procedure DS, in- stent (mm) & $11.4 \pm 8.1$ & $18.0 \pm 11.2$ & $<0.001$ \\
\hline Post-procedure DS, in-segment $(\mathrm{mm})$ & $21.0 \pm 11.3$ & $29.9 \pm 13.6$ & $<0.001$ \\
\hline
\end{tabular}


TABLE 2: Baseline patients and lesion characteristics according to late TLR.

\begin{tabular}{|c|c|c|c|}
\hline Clinical Factors & $\begin{array}{c}\text { Late TLR(-) } \\
\text { (335 patients) }\end{array}$ & $\begin{array}{l}\text { Late TLR }(+) \\
(19 \text { patients) }\end{array}$ & $\mathrm{P}$ \\
\hline Age (years old) & $65.5 \pm 10.3$ & $62.8 \pm 11.2$ & 0.272 \\
\hline BMI $\left(\mathrm{kg} / \mathrm{m}^{2}\right)$ & $24.9 \pm 2.9$ & $24.3 \pm 2.8$ & 0.429 \\
\hline Male sex, n (\%) & $249(74.3)$ & $16(84.2)$ & 0.488 \\
\hline Previous PCI, n (\%) & $33(9.9)$ & $5(26.3)$ & 0.061 \\
\hline Diabetes mellitus, n (\%) & $108(32.2)$ & $6(31.6)$ & 1.000 \\
\hline Hypertension, n (\%) & $232(69.3)$ & $14(73.7)$ & 0.879 \\
\hline CRF, n (\%) & $129(38.5)$ & $8(42.1)$ & 0.811 \\
\hline Dyslipidemia, n (\%) & $213(63.6)$ & $12(63.2)$ & 1.000 \\
\hline Current smoking, n (\%) & $87(26.0)$ & $8(42.1)$ & 0.201 \\
\hline FHx of CAD, n (\%) & $44(13.1)$ & $0(0)$ & 0.223 \\
\hline LV ejection fraction (\%) & $60.2 \pm 8.4$ & $62.5 \pm 6.9$ & 0.300 \\
\hline Clinical diagnosis & & & 0.632 \\
\hline (i) Silent ischemia, $\mathrm{n}(\%)$ & $19(5.7)$ & $2(10.5)$ & \\
\hline (ii) Stable angina, $\mathrm{n}(\%)$ & $197(58.8)$ & $12(63.2)$ & \\
\hline (iii) Unstable angina, $\mathrm{n}(\%)$ & $67(20.0)$ & $4(21.1)$ & \\
\hline (iv) STEMI, n (\%) & $21(6.3)$ & $1(5.3)$ & \\
\hline (v) NSTEMI, n (\%) & $31(9.3)$ & $0(0.0)$ & \\
\hline Multi Vessel disease, n (\%) & $245(73.1)$ & $10(52.6)$ & 0.066 \\
\hline \multicolumn{4}{|l|}{ Laboratory tests } \\
\hline $\mathrm{WBC}\left(10^{9} / \mathrm{L}\right)$ & $6.9 \pm 2.4$ & $7.4 \pm 2.2$ & 0.354 \\
\hline Hemoglobin (g/dl) & $13.6 \pm 1.8$ & $13.7 \pm 2.0$ & 0.840 \\
\hline Creatinine(mg/dl) & $1.04 \pm 0.43$ & $1.19 \pm 0.63$ & 0.303 \\
\hline $\operatorname{GFR}\left(\mathrm{ml} / \mathrm{min} / 1.73 \mathrm{~m}^{2}\right)$ & $70.9 \pm 24.1$ & $69.6 \pm 24.4$ & 0.864 \\
\hline Total cholesterol (mg/dl) & $157 \pm 38$ & $157 \pm 44$ & 0.982 \\
\hline Triglyceride (mg/dl) & $141 \pm 88$ & $118 \pm 38$ & 0.283 \\
\hline $\mathrm{LDL}(\mathrm{mg} / \mathrm{dl})$ & $97 \pm 35$ & $102 \pm 38$ & 0.576 \\
\hline HDL (mg/dl) & $42 \pm 11$ & $39 \pm 8$ & 0.277 \\
\hline CRP (mg/dl) & $0.34 \pm 0.92$ & $1.44 \pm 3.27$ & 0.200 \\
\hline \multicolumn{4}{|l|}{ Medication at discharge } \\
\hline (i) Aspirin, n (\%) & $334(99.7)$ & $19(100.0)$ & 1.000 \\
\hline (ii) Clopidogrel, n (\%) & $334(99.7)$ & $19(100.0)$ & 1.000 \\
\hline (iii) DAPT, $\mathrm{n}(\%)^{\#}$ & $333(99.4)$ & $19(100.0)$ & 1.000 \\
\hline (vi) Beta blocker, n (\%) & $237(70.7)$ & $14(73.7)$ & 1.000 \\
\hline (v) Statin, n (\%) & $318(94.9)$ & $19(100.0)$ & 0.612 \\
\hline (vi) CCB, n (\%) & $66(19.7)$ & $9(47.4)$ & 0.008 \\
\hline (vii) ACEI, n (\%) & $82(24.5)$ & $3(15.8)$ & 0.581 \\
\hline (viii) ARB, n (\%) & $128(38.2)$ & $6(31.6)$ & 0.634 \\
\hline \multicolumn{4}{|l|}{ Medication at first follow-up } \\
\hline (i) Aspirin, n (\%) & $332(99.1)$ & $19(100.0)$ & 1.000 \\
\hline (ii) Clopidogrel, n (\%) & $328(97.9)$ & $18(94.7)$ & 0.360 \\
\hline (iii) DAPT, n $(\%)^{\#}$ & $325(97.0)$ & $18(94.7)$ & 0.460 \\
\hline (vi) Beta blocker, n (\%) & $252(75.2)$ & $14(73.7)$ & 1.000 \\
\hline (v) Statin, n (\%) & $333(99.4)$ & $19(100.0)$ & 1.000 \\
\hline (vi) CCB, n (\%) & $72(21.5)$ & $7(36.8)$ & 0.153 \\
\hline (vii) ACEI, n (\%) & $35(10.4)$ & $1(5.3)$ & 0.706 \\
\hline (viii) ARB, n (\%) & $136(40.6)$ & $9(47.4)$ & 0.634 \\
\hline \multicolumn{4}{|l|}{ Medication at second follow-up } \\
\hline (i) Aspirin, n (\%) & $307(91.6)$ & $17(89.5)$ & 0.669 \\
\hline (ii) Clopidogrel, n (\%) & $282(84.2)$ & $18(94.7)$ & 0.329 \\
\hline (iii) DAPT, n (\%) & $256(76.4)$ & $16(84.2)$ & 0.581 \\
\hline
\end{tabular}


TABLe 2: Continued.

\begin{tabular}{|c|c|c|c|}
\hline Clinical Factors & $\begin{array}{l}\text { Late TLR(-) } \\
\text { (335 patients) }\end{array}$ & $\begin{array}{l}\text { Late } \operatorname{TLR}(+) \\
\text { (19 patients) }\end{array}$ & $\mathrm{P}$ \\
\hline (vi) Beta blocker, n (\%) & $244(72.8)$ & $12(63.2)$ & 0.429 \\
\hline (v) Statin, n (\%) & $330(98.5)$ & $19(100.0)$ & 1.000 \\
\hline (vi) CCB, n (\%) & $62(18.5)$ & $6(31.6)$ & 0.225 \\
\hline (vii) ACEI, n (\%) & $28(8.4)$ & $0(0)$ & 0.382 \\
\hline (viii) ARB, n (\%) & $127(37.9)$ & $10(52.6)$ & 0.230 \\
\hline \multirow[t]{2}{*}{ Lesion factors } & Late TLR(-) & Late TLR(+) & \\
\hline & (863 lesions) & (23 lesions) & \\
\hline Treated coronary location & & & 0.482 \\
\hline (i) LAD, n (\%) & $397(46.0)$ & $14(60.9)$ & \\
\hline (ii) LCX, n (\%) & $215(24.9)$ & $3(13.0)$ & \\
\hline (iii) RCA, n (\%) & $249(28.9)$ & $6(26.1)$ & \\
\hline Bifurcation lesion, n (\%) & $298(34.5)$ & $10(43.5)$ & 0.382 \\
\hline Calcified lesion & & & 0.109 \\
\hline (i) None, n (\%) & 437 (50.9) & $14(60.9)$ & \\
\hline (ii) Mild, n (\%) & $154(17.9)$ & $1(4.3)$ & \\
\hline (iii) Moderate, n (\%) & $113(13.2)$ & $1(4.3)$ & \\
\hline (vi) Severe, n (\%) & $154(17.9)$ & $7(30.4)$ & \\
\hline Tortuous lesion & & & 0.761 \\
\hline (i) None, n (\%) & $797(92.8)$ & $22(95.7)$ & \\
\hline (ii) Mild, n (\%) & $24(2.8)$ & $0(0)$ & \\
\hline (iii) Moderate, n (\%) & $25(2.9)$ & $1(4.3)$ & \\
\hline (vi) Severe, $\mathrm{n}(\%)$ & $13(1.5)$ & $0(0)$ & \\
\hline Angulation lesion, n (\%) & & & 0.428 \\
\hline (i) None $\left(<45^{\circ}\right), \mathrm{n}(\%)$ & $798(93.1)$ & $23(100.0)$ & \\
\hline (ii) Moderate $\left(45^{\circ}<90^{\circ}\right), \mathrm{n}(\%)$ & $37(4.3)$ & $0(0)$ & \\
\hline (iii) Extreme n $\left(>90^{\circ}\right), \mathrm{n}(\%)$ & $22(2.6)$ & $0(0)$ & \\
\hline Thrombus in lesion, $\mathrm{n}(\%)$ & $26(3.0)$ & $3(13.0)$ & 0.291 \\
\hline Ostial lesion, n (\%) & $165(19.1)$ & $4(17.4)$ & 1.000 \\
\hline Ulceration, n (\%) & $2(0.2)$ & $0(0)$ & 1.000 \\
\hline Aneurysm, n (\%) & $10(1.2)$ & $0(0)$ & 1.000 \\
\hline Lesion type & & & 0.132 \\
\hline (i) A, n (\%) & $87(10.1)$ & $5(21.7)$ & \\
\hline (ii) $\mathrm{B} 1, \mathrm{n}(\%)$ & $206(23.9)$ & $8(34.8)$ & \\
\hline (iii) $\mathrm{B} 2, \mathrm{n}(\%)$ & $50(5.8)$ & $2(8.7)$ & \\
\hline (vi) C, n (\%) & $491(56.9)$ & $8(34.8)$ & \\
\hline Type B2/C lesion, n (\%) & $541(64.9)$ & $10(43.5)$ & 0.059 \\
\hline Reference vessel diameter (mm) & $2.93 \pm 0.51$ & $2.89 \pm 0.53$ & 0.713 \\
\hline Lesion length (mm) & $27.7 \pm 17.1$ & $25.3 \pm 20.2$ & 0.561 \\
\hline Pre-procedure MLD (mm) & $0.74 \pm 0.50$ & $0.75 \pm 0.59$ & 0.926 \\
\hline Pre-procedure DS (\%) & $74.7 \pm 15.7$ & $74.9 \pm 17.3$ & 0.938 \\
\hline Post-procedure MLD, in- stent (mm) & $2.49 \pm 0.43$ & $2.55 \pm 0.40$ & 0.509 \\
\hline Post-procedure MLD, in-segment (mm) & $2.12 \pm 0.51$ & $2.26 \pm 0.55$ & 0.216 \\
\hline Post-procedure DS, in- stent (mm) & $11.4 \pm 8.1$ & $11.3 \pm 9.6$ & 0.955 \\
\hline Post-procedure DS, in-segment (mm) & $21.0 \pm 11.3$ & $20.5 \pm 10.9$ & 0.825 \\
\hline $\begin{array}{l}\text { TLR, target lesion revascularization; BMI, bod } \\
\text { history; CAD, coronary artery disease; LV, left v } \\
\text { white blood cell; GFR, glomerular filtration rat } \\
\text { DAPT, dual antiplatelet therapy; CCB, calcium } \\
\text { left anterior descending; LCX, left circumflex; }\end{array}$ & $\begin{array}{l}\text { protein cholester } \\
\text { angiotensin con } \\
\text { ery; MLD, minir }\end{array}$ & $\begin{array}{l}\text { ention; CRF, ch } \\
\text { non-ST-elevatio } \\
\text { poprotein chole } \\
\text { rs; ARB, angiote } \\
\text {, diameter steno }\end{array}$ & $\begin{array}{l}\text { family } \\
\text {; WBC, } \\
\text { protein; } \\
\text { s; LAD, }\end{array}$ \\
\hline
\end{tabular}


TABLE 3: Predictors of early TLR.

\begin{tabular}{|c|c|c|c|}
\hline Clinical Factors & Adjusted Hazard Ratio & $95 \% \mathrm{CI}$ & $\mathrm{P}$ \\
\hline Age (years old) & 0.96 & $0.92-1.00$ & 0.070 \\
\hline BMI $\left(\geq 23 \mathrm{~kg} / \mathrm{m}^{2}\right)$ & 0.78 & $0.37-1.61$ & 0.494 \\
\hline Gender (male) & 0.58 & $0.26-1.30$ & 0.187 \\
\hline Previous PCI & 2.41 & $1.03-5.65$ & 0.043 \\
\hline Diabetes mellitus & 2.58 & $1.29-5.15$ & 0.007 \\
\hline Hypertension & 0.70 & $0.31-1.60$ & 0.397 \\
\hline CRF & 2.37 & $0.94-5.98$ & 0.068 \\
\hline Dyslipidemia & 0.56 & $0.27-1.14$ & 0.108 \\
\hline Current smoking & 0.92 & $0.37-2.30$ & 0.858 \\
\hline FHx of CAD & 0.98 & $0.35-2.71$ & 0.967 \\
\hline LV dysfunction $(<40 \%)$ & 2.01 & $0.41-9.76$ & 0.387 \\
\hline Multivessel disease & 1.20 & $0.52-2.81$ & 0.669 \\
\hline Hemoglobin (g/dl) & 0.94 & $0.77-1.16$ & 0.586 \\
\hline $\mathrm{CRP}(\mathrm{mg} / \mathrm{dl})$ & 1.03 & $0.73-1.44$ & 0.889 \\
\hline Type B2/C lesion & 1.46 & $0.64-3.37$ & 0.371 \\
\hline Reference vessel diameter (mm) & 0.77 & $0.44-1.35$ & 0.366 \\
\hline Lesion length (mm) & 0.99 & $0.97-1.01$ & 0.208 \\
\hline Pre-procedure DS (\%) & 1.01 & $0.98-1.04$ & 0.546 \\
\hline Post-procedure DS\%, in- stent (mm) & 1.08 & $1.05-1.11$ & $<0.001$ \\
\hline
\end{tabular}

TLR, target lesion revascularization; CI, confidence interval; BMI, body mass index; PCI, percutaneous coronary intervention; CRF, chronic renal failure; FHx, family history; CAD, coronary artery disease; LV, left ventricle; CRP, C-reactive protein; DS, diameter stenosis.

TABLE 4: Predictors of late TLR.

\begin{tabular}{|c|c|c|c|}
\hline Clinical Factors & Adjusted Hazard Ratio & $95 \% \mathrm{CI}$ & $\mathrm{P}$ \\
\hline Age (years old) & 0.97 & $0.90-1.04$ & 0.359 \\
\hline BMI $\left(\geq 23 \mathrm{~kg} / \mathrm{m}^{2}\right)$ & 1.68 & $0.40-6.94$ & 0.477 \\
\hline Gender (male) & 2.39 & $0.38-14.95$ & 0.351 \\
\hline Previous PCI & 9.43 & $2.58-34.52$ & 0.001 \\
\hline Diabetes mellitus & 1.56 & $0.47-5.21$ & 0.472 \\
\hline Hypertension & 0.87 & $0.25-3.11$ & 0.835 \\
\hline CRF & 1.45 & $0.30-7.04$ & 0.641 \\
\hline Dyslipidemia & 0.70 & $0.20-2.48$ & 0.579 \\
\hline Current smoking & 2.56 & $0.78-8.40$ & 0.122 \\
\hline FHx of CAD & NA & NA & 0.974 \\
\hline LV dysfunction $(<40 \%)$ & NA & NA & 0.988 \\
\hline Multivessel disease & 0.42 & $0.12-1.41$ & 0.159 \\
\hline Hemoglobin (g/dl) & 1.19 & $0.77-1.85$ & 0.429 \\
\hline $\mathrm{CRP}(\mathrm{mg} / \mathrm{dl})$ & 1.60 & $1.28-2.00$ & $<0.001$ \\
\hline Type B2/C lesion & 0.22 & $0.04-1.11$ & 0.067 \\
\hline Reference vessel diameter (mm) & 0.82 & $0.31-2.21$ & 0.696 \\
\hline Lesion length (mm) & 1.03 & 0.99-1.08 & 0.165 \\
\hline Pre-procedure DS (\%) & 0.97 & 0.94-1.08 & 0.142 \\
\hline Post-procedure DS\%, in- stent (mm) & 1.01 & $0.95-1.08$ & 0.703 \\
\hline
\end{tabular}

TLR, target lesion revascularization; CI, confidence interval; BMI, body mass index; PCI, percutaneous coronary intervention; CRF, chronic renal failure; FHx, family history; CAD, coronary artery disease; LV, left ventricle; CRP, C-reactive protein; DS, diameter stenosis.

excessive NIH, which greatly reduced the incidence of ISR, there was a late catch-up phenomenon [22]. Due to drugs and polymers, DES prolongs the healing time of endothelial compared to BMS. This may be the reason why the incidence of early and late events is different. This is also the main reason for prolonging DAPT duration after entering the DES era. However, the exact mechanisms of ISR in DES remain unclear. The possible mechanisms of restenosis after DES that can be considered at present are biological factors (drug resistance, hypersensitivity), mechanical factors (stent 
TABLE 5: Lesion characteristics and DS\% progression rate according to types of DESs.

\begin{tabular}{|c|c|c|c|c|c|c|}
\hline Clinical Factors & $\begin{array}{c}\text { Overall } \\
(\mathrm{N}=944)\end{array}$ & $\begin{array}{c}\text { CoCr-EES } \\
(\mathrm{N}=244)\end{array}$ & $\begin{array}{c}\text { ZES } \\
(\mathrm{N}=310)\end{array}$ & $\begin{array}{c}\text { BES } \\
(\mathrm{N}=166)\end{array}$ & $\begin{array}{c}\text { PtCr-EES } \\
(\mathrm{N}=224)\end{array}$ & $\mathrm{P}$ \\
\hline Reference vessel diameter (mm) & $2.92 \pm 0.52$ & $2.97 \pm 0.56$ & $2.91 \pm 0.52$ & $2.96 \pm 0.49$ & $2.85 \pm 0.48$ & 0.054 \\
\hline Lesion length (mm) & $27.73 \pm 17.25$ & $26.04 \pm 16.45$ & $28.97 \pm 17.98$ & $26.56 \pm 15.96$ & $28.93 \pm 18.05$ & 0.247 \\
\hline Pre-procedure MLD (mm) & $0.73 \pm 0.50$ & $0.76 \pm 0.53$ & $0.65 \pm 0.51$ & $0.87 \pm 0.44$ & $0.71 \pm 0.46$ & 0.755 \\
\hline \multicolumn{7}{|l|}{ MLD in-segment (mm) } \\
\hline (i) Post-procedure & $2.11 \pm 0.52$ & $2.13 \pm 0.55$ & $2.08 \pm 0.52$ & $2.13 \pm 0.50$ & $2.11 \pm 0.51$ & 0.906 \\
\hline (ii) $1^{\text {st }}$ follow-up & $1.97 \pm 0.58$ & $1.97 \pm 0.59$ & $1.99 \pm 0.57$ & $1.95 \pm 0.58$ & $1.96 \pm 0.58$ & 0.726 \\
\hline (iii) $2^{\text {nd }}$ follow-up & $1.96 \pm 0.52$ & $2.01 \pm 0.55$ & $1.94 \pm 0.52$ & $1.96 \pm 0.49$ & $1.93 \pm 0.52$ & 0.180 \\
\hline \multicolumn{7}{|l|}{ MLD in-stent (mm) } \\
\hline (i) Post-procedure & $2.48 \pm 0.44$ & $2.49 \pm 0.47$ & $2.47 \pm 0.43$ & $2.47 \pm 0.42$ & $2.48 \pm 0.44$ & 0.852 \\
\hline (ii) $1^{\text {st }}$ follow-up & $2.19 \pm 0.60$ & $2.19 \pm 0.62$ & $2.20 \pm 0.59$ & $2.21 \pm 0.59$ & $2.15 \pm 0.60$ & 0.782 \\
\hline (iii) $2^{\text {nd }}$ follow-up & $2.13 \pm 0.55$ & $2.16 \pm 0.61$ & $2.12 \pm 0.54$ & $2.17 \pm 0.50$ & $2.09 \pm 0.54$ & 0.480 \\
\hline Pre-procedure DS (\%) & $74.8 \pm 15.7$ & $74.4 \pm 16.0$ & $77.0 \pm 16.6$ & $70.6 \pm 13.7$ & $75.1 \pm 14.9$ & 0.431 \\
\hline \multicolumn{7}{|l|}{ DS\% in-segment } \\
\hline (i) Post-procedure & $21.5 \pm 11.6$ & $22.9 \pm 12.5$ & $21.1 \pm 11.8$ & $21.4 \pm 10.8$ & $20.8 \pm 11.0$ & 0.075 \\
\hline (ii) $1^{\text {st }}$ follow-up & $26.2 \pm 16.5$ & $27.3 \pm 17.0$ & $25.0 \pm 15.9$ & $27.5 \pm 17.6$ & $25.8 \pm 15.9$ & 0.649 \\
\hline (iii) $2^{\text {nd }}$ follow-up & $27.3 \pm 15.0$ & $27.9 \pm 15.8$ & $26.2 \pm 15.3$ & $27.7 \pm 12.6$ & $28.1 \pm 15.2$ & 0.624 \\
\hline \multicolumn{7}{|l|}{ DS\% in-stent } \\
\hline (i) Post-procedure & $11.8 \pm 8.5$ & $13.0 \pm 8.4$ & $10.5 \pm 8.8$ & $12.6 \pm 9.0$ & $11.7 \pm 7.4$ & 0.460 \\
\hline (ii) $1^{\text {st }}$ follow-up & $22.0 \pm 17.5$ & $22.4 \pm 17.9$ & $20.8 \pm 16.4$ & $21.8 \pm 18.3$ & $23.3 \pm 17.9$ & 0.454 \\
\hline (iii) $2^{\text {nd }}$ follow-up & $23.7 \pm 15.5$ & $24.0 \pm 17.8$ & $22.8 \pm 15.3$ & $22.9 \pm 12.3$ & $25.3 \pm 15.5$ & 0.346 \\
\hline \multicolumn{7}{|l|}{ Acute gain $(\mathrm{mm})$} \\
\hline (i) In-segment & $1.38 \pm 0.59$ & $1.37 \pm 0.58$ & $1.42 \pm 0.63$ & $1.26 \pm 0.48$ & $1.40 \pm 0.60$ & 0.731 \\
\hline (ii) In-stent & $1.74 \pm 0.54$ & $1.73 \pm 0.53$ & $1.81 \pm 0.58$ & $1.61 \pm 0.47$ & $1.75 \pm 0.51$ & 0.395 \\
\hline $\begin{array}{l}\text { Initial } \Delta D S \\
\text { In-segment (\%) }\end{array}$ & $4.85 \pm 15.13$ & $4.71 \pm 16.53$ & $4.00 \pm 14.80$ & $6.33 \pm 14.32$ & $5.10 \pm 14.59$ & 0.457 \\
\hline $\begin{array}{l}\text { Initial } \Delta \mathrm{DS} \\
\text { In-stent (\%) }\end{array}$ & $10.28 \pm 15.89$ & $9.52 \pm 16.02$ & $10.27 \pm 15.15$ & $9.46 \pm 14.43$ & $11.72 \pm 17.63$ & 0.204 \\
\hline $\begin{array}{l}\text { Delayed } \Delta \mathrm{DS} \\
\text { In-segment (\%) }\end{array}$ & $3.66 \pm 11.87$ & $3.65 \pm 13.52$ & $2.82 \pm 11.53$ & $4.04 \pm 9.95$ & $4.55 \pm 11.81$ & 0.264 \\
\hline $\begin{array}{l}\text { Delayed } \Delta \mathrm{DS} \\
\text { In-stent }(\%)\end{array}$ & $4.59 \pm 11.60$ & $4.81 \pm 14.08$ & $3.73 \pm 9.87$ & $5.27 \pm 11.49$ & $5.10 \pm 11.11$ & 0.486 \\
\hline Initial DS\%/year in-segment & $6.08 \pm 20.77$ & $6.48 \pm 22.87$ & $4.75 \pm 17.49$ & $10.22 \pm 29.61$ & $4.45 \pm 13.00$ & 0.841 \\
\hline Initial DS\%/year in-stent & $12.12 \pm 20.97$ & $12.23 \pm 20.92$ & $12.15 \pm 18.60$ & $14.34 \pm 29.76$ & $10.36 \pm 15.74$ & 0.525 \\
\hline Delayed DS\%/year in-segment & $2.98 \pm 9.84$ & $2.65 \pm 9.93$ & $2.09 \pm 9.57$ & $3.26 \pm 7.89$ & $4.30 \pm 11.16$ & 0.130 \\
\hline Delayed DS\%/year in-stent & $3.68 \pm 10.10$ & $4.11 \pm 12.83$ & $2.39 \pm 8.13$ & $4.02 \pm 8.30$ & $4.77 \pm 10.46$ & 0.205 \\
\hline
\end{tabular}

CoCr-EES, cobalt chromium everolimus-eluting stent; ZES, zotarolimus-eluting stent; BES, Biolimus-eluting stent: PtCr-EES, platinum chromium everolimuseluting stent; MLD, minimal lumen diameter; DS, diameter stenosis.

underexpansion, nonuniform stent strut distribution, stent fracture, nonuniform drug elution/deposition, and polymer peeling), and technical factors (barotrauma outside stented segment, stent gap, and residual uncovered atherosclerotic plaques) [22]) DESs are constantly improving, but ISR is still an important difficulty to overcome.

4.2. Predictors of In-Stent Restenosis. The "late catch-up" phenomenon mentioned by previous studies suggests that the mechanism and rate of neointimal formation may be different at different time intervals after the 1st generation DES implantation [8-14]. After implantation of DES, the stent will gradually be covered by neointima, which may last for about one year due to the effect of the drug and polymer. Assuming that one year is the benchmark, the predictors may be different within one year and one year later. In our study, the incidence of early TLR in our study was $6.1 \%$, while the rate of late TLR was $2.4 \%$. Our data also showed that independent predictors for late TLR differed from the predictors of early TLR. Previous studies had suggested that the predictors of early TLR are MLD poststent implantation, saphenous vein graft, DM, RCA disease, family history of $\mathrm{CAD}$, multivessel disease, stent diameter, etc., while insulintreated DM, younger age, elevated serum hs-CRP levels, the 
first generation DES, stent fracture, stent diameter, and stent length are predictive predictors of late TLR [16, 23-25]. Some studies focused on early TLR which was evaluated at 8-12 months after PCI $[25,26]$, while other studies focused on late TLR, which was evaluated at 12-24 months after index PCI $[23,24]$. Due to the distinct characteristics of different cohorts, this should be analyzed in an identical cohort to compare the different predictors of TLR at different points in time. Also, most of the stents in these studies are first generation DES, which are known to have a high rate of TLR compared to that with contemporary DESs $[26,27]$. With the constant improvement of DES, the predictors of ISR may be different in new generation DES. Compared to the above studies, the population of our study was all treated with 2nd generation DES, and both the predictors of early and late TLR were analyzed in a single cohort. From the result of our study, the independent predictors of early TLR were previous PCI, DM, and postprocedure DS, while previous PCI and CRP were independent predictors for late TLR. The predictors in our study can be explained as follows. A history of PCI, which was a consistent predictor of early and late TLR, is known to directly represent the overall atherosclerotic risk of an individual and is a general risk factor for vascular atherosclerosis and restenosis [28]. As for $\mathrm{DM}$, some putative mechanisms, including more aggressive intimal hyperplasia, higher inflammatory response, higher coagulability, and endothelial dysfunction, have been considered to be probable causes of a high rate of early TLR in patients with DM after stent implantation [29]. Procedural factors also determine restenosis, such as stent underexpansion and incomplete apposition. In our study, postprocedural DS was only a predictor of early TLR, which could explain why procedural factors are important to prevent restenosis in the early phase. CRP is recognized as an important marker of vascular wall inflammation and as a strong predictor of adverse cardiovascular events. It may play an important role in the pathogenesis of NIH after coronary stenting [30]. Choi et al.'s [16] study also suggested that high levels of CRP is an independent risk factor for late TLR after DES implantation. Our findings are on the same line as this study. High CRP levels mean chronic inflammatory state and promote $\mathrm{NIH}$, which can persist to late stages and affect the ISR progression.

Collectively, we analyzed the predictors of early and late TLR in patients treated with 2nd generation DES in a single cohort, which could discriminate the distinct predictors of TLR in different phases. Quantitative analysis for progression of ISR was also performed making our findings more convincing.

4.3. ISR Progression in 2nd Generation DES. For further analysis, we compared the ISR progression rate using QCA analysis. The early delta DS\%/year was 3.3-fold larger than the delayed delta DS\%/year, implying that ISR progression is more rapid in the early phase. Interestingly, a previous study by Kang et al. analyzed the natural progression of atherosclerotic plaques [31]. This study showed that the natural progression rate of nonstented lesion was 2.19\%/year, which was smaller than stented lesions. From these results, we can conclude that stented lesions are more susceptible to restenosis, compared to the progression of natural atherosclerotic lesions.

4.4. Limitation. Frist, due to the retrospective nature of our study, there could have been a selection bias in patient selection. We compared the baseline clinical characteristics with the total parent population whom received PCI during the study period, and we found minimal difference between the two populations. However, we cannot complete deny possibilities of other selection bias within our study population. Second, we did not perform imaging analysis using IVUS, which may enable assessment of the cause of restenosis. Lastly, the data used in analysis in this study were based on a relatively small sample and were collected from a single center.

\section{Conclusion}

Our data suggested that the factors of DM, previous PCI, and postprocedure in-stent DS\% were predictors of early TLR, and the predictors for late TLR were previous PCI and high level serum CRP. Predictors for TLR may be different at different time intervals in 2nd generation DES era. There was no difference in the rate of DS progression in stented lesion between four types of contemporary DESs.

\section{Data Availability}

The data used to support the findings of this study are available from the corresponding author upon request.

\section{Conflicts of Interest}

The authors declare that they have no conflicts of interest.

\section{Authors' Contributions}

Chengbin Zheng and Jeehoon Kang contributed equally to the study.

\section{Acknowledgments}

This research was supported by a research grant of the EXCELLENT-Prime study (Research ID: 06-2011-3680), HOST-Resolinte study (Research ID: 06-2011-3280), HOSTBiolimus study (Research ID: 06-2010-1560), Endocor Korea study (Research ID: 800-2015-0069), and MR-KOPRE study (Research ID: 06-2011-2830). Sponsors had no role in the study design or data analysis and interpretation or in the decision to submit the manuscript for publication.

\section{Supplementary Materials}

Figure 1. Cumulative probability curve of MLD (postprocedure, first angiographic follow-up, and second angiographic follow-up). Table 1. Baseline demographic and clinical characteristics of the current study population and total patent 
population. Table 2. Baseline patients and lesions characteristics. Table 3. Laboratory findings at the follow-up periods. Table 4. Clinical events during the follow-up period. Table 5. Ischemic driven and angiographic driven TLR. Table 6. TLR method. (Supplementary Materials)

\section{References}

[1] R. Komatsu, M. Ueda, T. Naruko, A. Kojima, and A. E. Becker, "Neointimal tissue response at sites of coronary stenting in humans: macroscopic, histological, and immunohistochemical analyses," Circulation, vol. 98, no. 3, pp. 224-233, 1998.

[2] R. Hoffmann, G. S. Mintz, G. R. Dussaillant et al., "Patterns and mechanisms of in-stent restenosis," Circulation, vol. 94, no. 6, pp. 1247-1254, 1996.

[3] H. Mudra, E. Regar, V. Klauss et al., "Serial follow-up after optimized ultrasound-guided deployment of palmaz-schatz stents," Circulation, vol. 95, no. 2, pp. 363-370, 1997.

[4] N. Kuroda, Y. Kobayashi, M. Nameki et al., "Intimal hyperplasia regression from 6 to 12 months after stenting," American Journal of Cardiology, vol. 89, no. 7, pp. 869-872, 2002.

[5] T. Kimura, H. Yokoi, Y. Nakagawa et al., "Three-year follow-up after implantation of metallic coronary-artery stents," The New England Journal of Medicine, vol. 334, no. 9, pp. 561-566, 1996.

[6] G. W. Stone, S. G. Ellis, D. A. Cox et al., "A polymer-based, paclitaxel-eluting stent in patients with coronary artery disease," The New England Journal of Medicine, vol. 350, no. 3, pp. 221-231, 2004.

[7] J. W. Moses, M. B. Leon, J. J. Popma et al., "Sirolimus-eluting stents versus standard stents in patients with stenosis in a native coronary artery," The New England Journal of Medicine, vol. 349, no. 14, pp. 1315-1323, 2003.

[8] K. W. Park, C.-H. Kim, H.-Y. Lee et al., "Does 'late catch-up' exist in drug-eluting stents: Insights from a serial quantitative coronary angiography analysis of sirolimus versus paclitaxeleluting stents," American Heart Journal, vol. 159, no. 3, pp. 446.e443-453.e443, 2010.

[9] R. A. Byrne, R. Iijima, J. Mehilli et al., "Durability of antirestenotic efficacy in drug-eluting stents with and without permanent polymer," JACC: Cardiovascular Interventions, vol. 2, no. 4, pp. 291-299, 2009.

[10] R. Wessely, A. Kastrati, and A. Schömig, "Late restenosis in patients receiving a polymer-coated sirolimus-eluting stent," Annals of Internal Medicine, vol. 143, no. 5, pp. 392-394, 2005.

[11] M. Valgimigli, P. Malagutti, C. A. van Mieghem et al., "Persistence of neointimal growth 12 months after intervention and occurrence of delayed restenosis in patients with left main coronary artery disease treated with drug-eluting stents," Journal of the American College of Cardiology, vol. 47, no. 7, pp. 1491-1494, 2006.

[12] I. Sheiban, A. Chiribiri, S. Galli et al., "Sirolimus-eluting stent implantation for bare-metal in-stent restenosis: is there any evidence for a late catch-up phenomenon?" Journal of Cardiovascular Medicine, vol. 9, no. 8, pp. 783-788, 2008.

[13] D. Park, M. Hong, G. S. Mintz et al., "Two-year follow-up of the quantitative angiographic and volumetric intravascular ultrasound analysis after nonpolymeric paclitaxel-eluting stent implantation," Journal of the American College of Cardiology, vol. 48, no. 12, pp. 2432-2439, 2006.
[14] A. V. Finn, G. Nakazawa, M. Joner et al., "Vascular responses to drug eluting stents: importance of delayed healing," Arteriosclerosis, Thrombosis, and Vascular Biology, vol. 27, no. 7, pp. 1500$1510,2007$.

[15] Y. Nakagawa, T. Kimura, T. Morimoto et al., "Incidence and risk factors of late target lesion revascularization after sirolimuseluting stent implantation (3-year follow-up of the j-cypher registry)," American Journal of Cardiology, vol. 106, no. 3, pp. 329-336, 2010.

[16] I. J. Choi, H. Park, S. Seo et al., "Predictors of early and late target lesion revascularization after drug-eluting stent implantation," Journal of Interventional Cardiology, vol. 26, no. 2, pp. 137-144, 2013.

[17] D. Buccheri, D. Piraino, G. Andolina, and B. Cortese, "Understanding and managing in-stent restenosis: a review of clinical data, from pathogenesis to treatment," Journal of Thoracic Disease, vol. 8, no. 10, pp. E1150-E1162, 2016.

[18] R. Virmani, F. Liistro, G. Stankovic et al., "Mechanism of late in-stent restenosis after implantation of a paclitaxel derivateeluting polymer stent system in humans," Circulation, vol. 106, no. 21, pp. 2649-2651, 2002.

[19] I. Goto, T. Itoh, T. Kimura et al., "Morphological and quantitative analysis of vascular wall and neointimal hyperplasia after coronary stenting," Circulation Journal, vol. 75, no. 7, pp. 16331640, 2011.

[20] S. J. Nicholls, E. M. Tuzcu, I. Sipahi et al., "Statins, highdensity lipoprotein cholesterol, and regression of coronary atherosclerosis," Journal of the American Medical Association, vol. 297, no. 5, pp. 499-508, 2007.

[21] K. Goto, Z. Zhao, M. Matsumura et al., "Mechanisms and patterns of intravascular ultrasound in-stent restenosis among bare metal stents and first- and second-generation drug-eluting stents," American Journal of Cardiology, vol. 116, no. 9, pp. 13511357, 2015.

[22] M. S. Lee and G. Banka, "In-stent restenosis," Interventional Cardiology Clinics, vol. 5, no. 2, pp. 211-220, 2016.

[23] H. Hara, J. Aoki, K. Tanabe et al., "Incidence and predictors for late target lesion revascularization after sirolimus-eluting stent implantation," Circulation Journal, vol. 77, no. 4, pp. 988-994, 2013.

[24] M. Hong, G. S. Mintz, C. W. Lee et al., "Late target lesion revascularization after implantation of sirolimus-eluting stent," Catheterization and Cardiovascular Interventions, vol. 71, no. 3, pp. 299-303, 2008.

[25] Y. Takasawa, R. Iijima, M. Shiba, M. Nakamura, and K. Sugi, "Predictor of subsequent target lesion revascularization in patients with drug-eluting stent restenosis undergoing percutaneous coronary intervention," Journal of Cardiology, vol. 55, no. 3, pp. 391-396, 2010.

[26] Y. Watanabe, K. Takagi, T. Naganuma et al., "Independent predictors of in-stent restenosis after drug-eluting stent implantation for ostial right coronary artery lesions," International Journal of Cardiology, vol. 240, pp. 108-113, 2017.

[27] R. Piccolo, T. Pilgrim, D. Heg et al., "Comparative effectiveness and safety of new-generation versus early-generation drugeluting stents according to complexity of coronary artery disease," JACC: Cardiovascular Interventions, vol. 8, no. 13, pp. 1657-1666, 2015.

[28] D. A. Morrow, "Cardiovascular risk prediction in patients with stable and unstable coronary heart disease," Circulation, vol. 121, no. 24, pp. 2681-2691, 2010. 
[29] S. C. Smith, D. Faxon, W. Cascio et al., "Prevention conference VI: diabetes and cardiovascular disease: writing group VI: revascularization in diabetic patients," Circulation, vol. 105, no. 18, pp. e165-e169, 2002.

[30] P. M. Ridker, "Clinical application of C-reactive protein for cardiovascular disease detection and prevention," Circulation, vol. 107, no. 3, pp. 363-369, 2003.

[31] J. Kang, K. W. Park, M. S. Lee et al., "The natural course of nonculprit coronary artery lesions; analysis by serial quantitative coronary angiography," BMC Cardiovascular Disorders, vol. 18, no. 1, p. 130, 2018. 


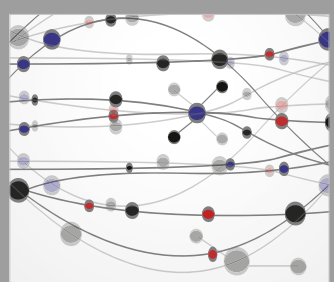

The Scientific World Journal
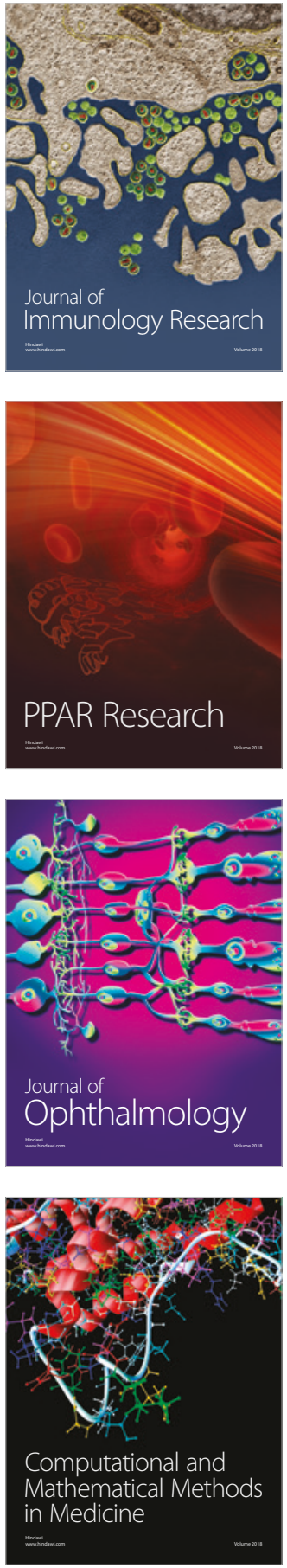

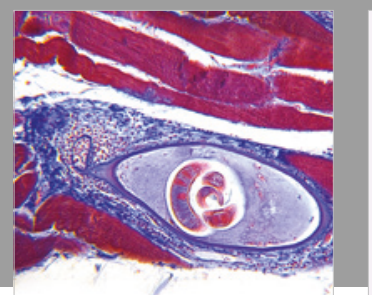

Gastroenterology Research and Practice

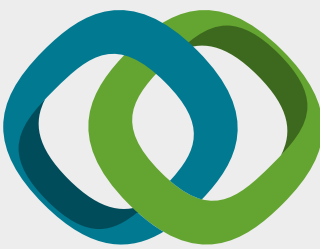

\section{Hindawi}

Submit your manuscripts at

www.hindawi.com
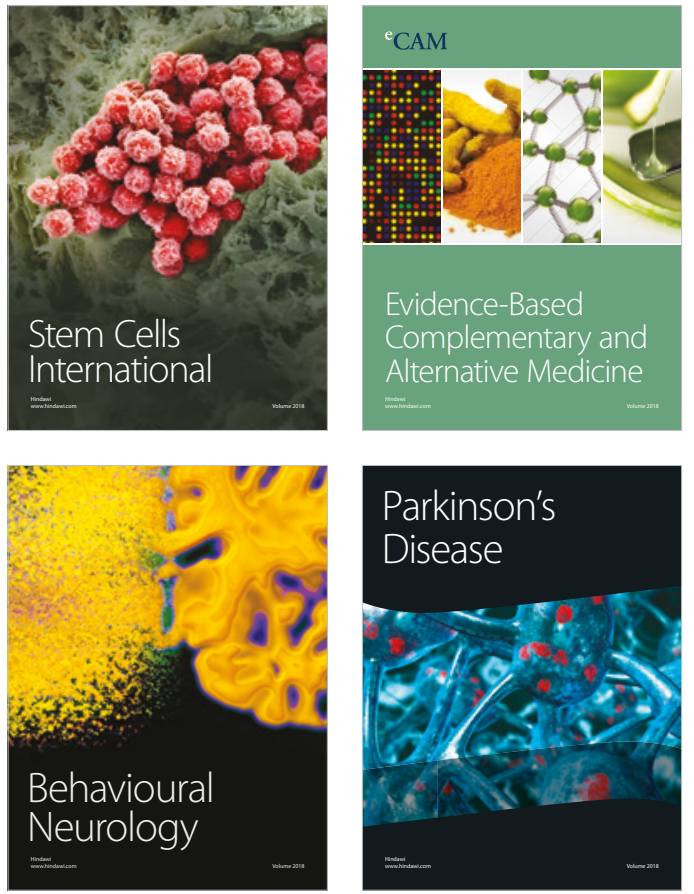

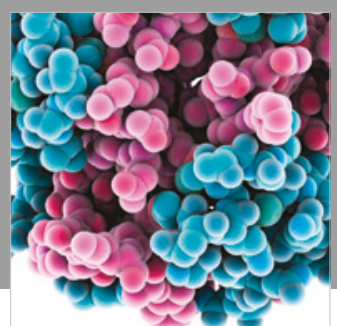

ournal of

Diabetes Research

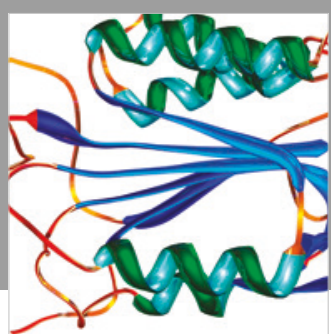

Disease Markers
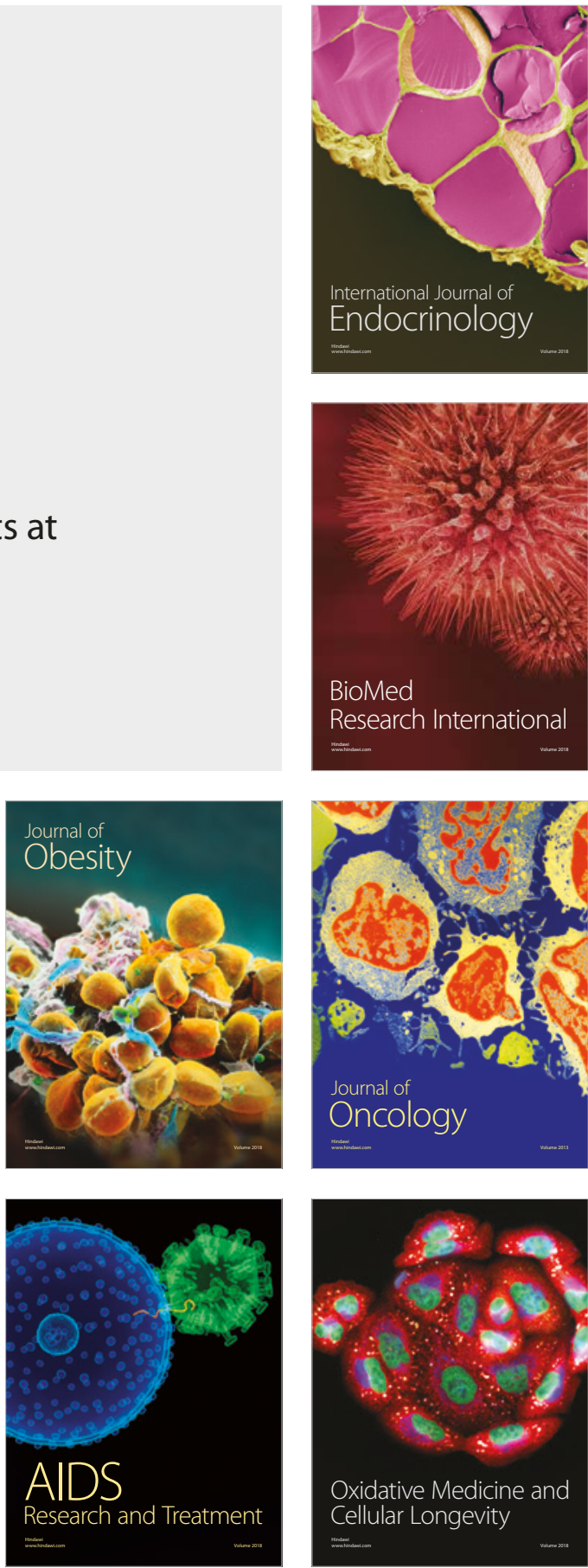